\title{
VAB and MRI Following Percutaneous Ultra-Sound Guided Cryoablation for Primary Early-Stage Breast Cancer: A Pilot Study in Japan
}

\author{
Hisanori Kawamoto1,2*, Koichiro Tsugawa',2, Mariko Takishita², Mizuho Tazo ${ }^{2}$, \\ Mari Hara Nakano², Tomoko Tsuruga ${ }^{2}$, Ryoko Oi $^{2}$, Takako Kuroda' ${ }^{2}$, Reiko Yoshie ${ }^{2}$, \\ Ayaka Shimo', Tsuguo Iwatani2, Arata Shimo², Yasuyuki Kojima², Ai Motoyoshi2, Ei Haku',2, \\ Yukari Yabuki², Akihiko Suto1, Yoshihide Kanemaki1,3, Hidefumi Mimura³, Ichiro Maeda", \\ Mamoru Fukuda1
}

\begin{abstract}
${ }^{1}$ Department of Breast Surgery Breast and Imaging Center, St. Marianna University School of Medicine, Kawasaki-Shi, Japan ${ }^{2}$ Division of Breast and Endocrine Surgery, Department of Surgery, St. Marianna University School of Medicine, Kawasaki-Shi, Japan

${ }^{3}$ Department of Radiology, St. Marianna University School of Medicine, Kawasaki-Shi, Japan

${ }^{4}$ Department of Pathology, St. Marianna University School of Medicine, Kawasaki-Shi, Japan

Email: ^h-kawamoto@marianna-u.ac.jp
\end{abstract}

How to cite this paper: Kawamoto, H., Tsugawa, K., Takishita, M., Tazo, M., Nakano, M.H., Tsuruga, T., Oi, R., Kuroda, T., Yoshie, R., Shimo, A., Iwatani, T., Shimo, A., Kojima, Y., Motoyoshi, A., Haku, E., Yabuki, Y., Suto, A., Kanemaki, Y., Mimura, H., Maeda, I. and Fukuda, M. (2021) VAB and MRI Following Percutaneous Ultra-Sound Guided Cryoablation for Primary Early-Stage Breast Cancer: A Pilot Study in Japan. Journal of Cancer Therapy, 12, 563-576.

https://doi.org/10.4236/jct.2021.1210047

Received: September 19, 2021

Accepted: October 23, 2021

Published: October 26, 2021

Copyright $\odot 2021$ by author(s) and Scientific Research Publishing Inc. This work is licensed under the Creative Commons Attribution International License (CC BY 4.0).

http://creativecommons.org/licenses/by/4.0/

cc) (i) Open Access

\begin{abstract}
Objective: This study aimed to evaluate the feasibility, safety, and cosmetic outcomes of percutaneous cryoablation (PCA) of breast intraductal carcino$\mathrm{ma}$ (IDC) lesions, as well as post-cryoablation magnetic resonance imaging (MRI) as a follow-up tool for detection of residual malignancies and local recurrences. Methods: Eight female patients underwent percutaneous ultrasoundguided cryoablation of breast IDC tumors under local anesthesia without subsequent resection. All patients received radiation- and endocrine therapies (RT, ET). The patients were followed using vacuum-assisted biopsy (VAB), mammography (MG), magnetic resonance imaging (MRI), and Moiré Topography for cosmetic outcomes. VAB was performed 6 months following cryoablation treatment for cases 1 and 2 (after starting radiation- and endocrine-therapies) or one month after PCA (cases 3, 4, 5, 6 and 7), prior to RT and ET. One patient declined VAB. Results: Mean age of the patients was 61.9 years, SD 7.7, ages range $53-72$ years. Mean tumor size was $10.3 \mathrm{~mm}$, SD 2.74, ranged $6.8-14.5 \mathrm{~mm}$, median follow-up time was 28 months (range 13 - 34 months). No residual or recurrent malignancies were detected. One minor adverse event was observed: A skin redness in the ablated area; MRI at one-month post-cryotherapy showed various degrees of thermal burns in all
\end{abstract}


patients in the pectoralis major muscle, which were not symptomatic and were resolved by 6 months following PCA. Decrease of fat necrosis areas in the vicinity of ablated ex-tumor was traced with MRI (mean size $54.9 \mathrm{~mm}$, mean decrease after 2 years was 58\%) and validated with VAB. Conclusions: Percutaneous cryoablation of early-stage low-risk breast cancer tumors smaller than $15 \mathrm{~mm}$ potentially presents a potential substitute for lumpectomy, offering encouraging short- to mid-term oncology results with good cosmesis outcomes. Patients would be able to benefit from local anesthesia in an outpatient setting and a shorter recovery period.

\section{Keywords}

Breast Cancer, Cryosurgery, Cryoablation, Cryotherapy, Day Surgery

\section{Introduction}

Breast cancer (BC) is the most common type of cancer in the world and the second most common type of cancer among women. The proportion of new worldwide cases of breast cancer to all types of cancer in women in 2020 was $21.4 \%$, followed by colorectal cancer (15.7\%), lung cancer (10.3\%), and other types of cancer. In 2018, there were 254,744 new cases of Female Breast Cancer in the United States, and 42,465 deaths [1].

In 2021, the National Institute of Health (NIH) predicted 281,550 new cases of female breast cancer and 43,600 deaths from this disease [2].

According to the World Health Organization (WHO), in Japan, the total number of new BC cases was 99,024 (8.9\% of all new cancer cases) and 17,081 deaths (4.1\% of all deaths) reported in 2020. Age-standardized incidence of BC was $76.3 / 100,000$ among Japanese women in 2020 [3].

In Japan, the age of breast cancer diagnosis is quite young. The peak age incidence of $\mathrm{BC}$ has been decreasing in Japan during the last decade and, today, it appears in two peaks: 45 - 49 and 60 - 64 years of age. For comparison, the peak of incidence in US falls on 75 - 79 years of age [1]. In their 40's and 50's, women in Japan, as well as in many other countries, actively advance their roles both in the society and the family, which defines a strong demand for a well-tolerated reliable treatment with fast recovery and uncompromised cosmetic outcomes. Breast cancer presents a substantial burden, not only for patients and their families but also for the society. In addition, the cost of illness (COI) of BC has increased in recent years [4].

Novel technologies, patients' awareness of breast cancers early detection methods as a standard of healthcare, and compliance to screening helps detect breast cancer at very early stages.

Although the golden standard approach to the treatment of localized breast cancer (breast-conserving surgery) provides a five years survival rate above $99 \%$ [5], while satisfying tolerability and local curability, it often involves undesirable 
side effects, such as pain, risks associated with surgical interventions, breast deformation and skin scars.

Several modern techniques for minimally invasive tumor ablation have been reported, including radiofrequency ablation (RFA), laser-ablation, cryoablation, high-intensity focused ultrasound (HIFU), microwave ablation, and, more recently, stereotactic radio-ablation in breast cancer [6]. Among others, cryoablation offers a minimally invasive solution to surgical resection: most notably, increased preservation of surrounding tissues, reduced cost and shorter hospitalization time [4]. The fact that a procedure can be limited to a day surgery under local anesthesia and provide good cosmetic outcomes, substantially decreases the psychological burden on the patients [7] [8] [9]. Progress in modern imaging and screening programs has led the method of minimally invasive thermal ablation of tumors to become commonly used [8]. Percutaneous cryoablation may be a good substitute for local excision in case of a small lesion and lack of axillary lymph nodes involvement [10] [11]队[12].

Precise patient selection criteria are of upmost importance for optimal navigation among currently offered breast cancer treatment options. Various studies underline that cryoablation is especially successful when treating small early-stage cancers up to $15 \mathrm{~mm}$. Takada et al. stressed that eligibility criteria for cryoablation include unifocal tumor, tumor size less than $2 \mathrm{~cm}, 1 \mathrm{~cm}$ distance from the skin surface, and lack of an in-situ component [9].

Large-scale prospective trials, such as "Cryoablation of Low-Risk Small Breast Cancer” (ICE3 Trial, NCT02200705) [13], "Cryoablation of Small Breast Tumors in Early-Stage Breast Cancer" (FROST, NCT01992250) [14] and "Cryoablation Therapy in Treating Patients with Invasive Ductal Breast Cancer" (ACOSOG Alliance Z1072, NCT00723294) [15] were designed for the patients with low-risk breast cancer of $15 \mathrm{~mm}$ or less. ACOSOG clinical trial has completed their activities and reported the results; ICE3 and FROST are still active. ICE3 clinical trial published their 3-year interim results this year. In our pilot study, we used inclusion criteria similar to that of ICE3 and FROST trials.

\section{Ethics Committee}

This study was approved by the Ethics Review Board of St. Marianna University (Approval number: No. 3627), registered with the UMIN clinical trials registry (UMIN000031222: Safety and effectiveness of non-resection ultrasound-guided cryotherapy for localized early breast cancer) and conducted at St. Marianna University Breast \& Imaging Center, Kawasaki City, Japan and. All patients provided written informed consent prior to the treatment.

\section{Methods}

Adult females of 20 - 85 years old diagnosed with first-onset invasive ductal carcinoma (IDC) of the breast, with Eastern Cooperative Oncology Group Performance Status of 0 or 1, hormone receptor-positive status, HER2 protein expres- 
sion-negative status, Ki-67 positivity $\leq 20 \%$, a unifocal primary lesion detected by mammography (MG), ultrasonography (US), and (or) magnetic resonance imaging (MRI), lesion spread of $15 \mathrm{~mm}$ or less, negative SLN biopsy results and amenability to radiation therapy, were included in the study.

Exclusion criteria were invasive lobular carcinoma, invasive micropapillary carcinoma, intraductal lesions, lesions located closer than $5 \mathrm{~mm}$ to the skin and pectoralis major muscle.

At the beginning of the cryoablation procedure, tumor location was marked under US guidance (UltraClip ${ }^{\mathrm{TM}}$ Dual Trigger Breast Tissue Markers or UltraCor $^{\mathrm{TM}}$ Twirl $^{\mathrm{TM}}$ Breast Marker (BD, C.R. Bard, Inc., Tempe, AZ, USA)). The skin incision site was chosen so that the cryoprobe tract would pass through the longest diameter of the lesion (Figure 1 and Figure 2). It was marked on the patient's chest with a skin marker.

Ultrasound guided percutaneous cryoablation was performed in outpatient clinic' premises under local anesthesia using liquid nitrogen-based device (ProSense ${ }^{\mathrm{TM}}$ cryoablation system, IceCure Medical $^{{ }^{\oplus}}$, Israel) and a single probe of 10 $\mathrm{G} / 140 \mathrm{~mm}$. Saline hydrodissection was employed to protect the skin or muscle during freezing. The elliptically shaped ice ball, produced by the cryoprobe, was repeatedly measured with US throughout the ablation procedure. We aimed to produce ice ball coverage of at least $35 \mathrm{~mm}$ to assure full coverage of the tumor with adequate margins. Wherever necessary, cryoablation cycles were repeated to obtain the necessary size of the ice ball.

All patients received whole breast irradiation $50 \mathrm{~Gy} / 25$ times and aromatase inhibitor as endocrine therapy.

We used vacuum-assisted biopsy (VAB), ultrasound (US), mammography (MG), and magnetic resonance imaging (MRI) at baseline, 1-, 6-, 12-, and 24-months post-ablation for follow up assessment of oncology outcomes. Seven of eight patients had US-guided VAB (patient no.8 refused post-ablation VAB) for confirmation of MG/MRI results (VAB device EnCorEnspire ${ }^{\oplus}$ Breast Biopsy

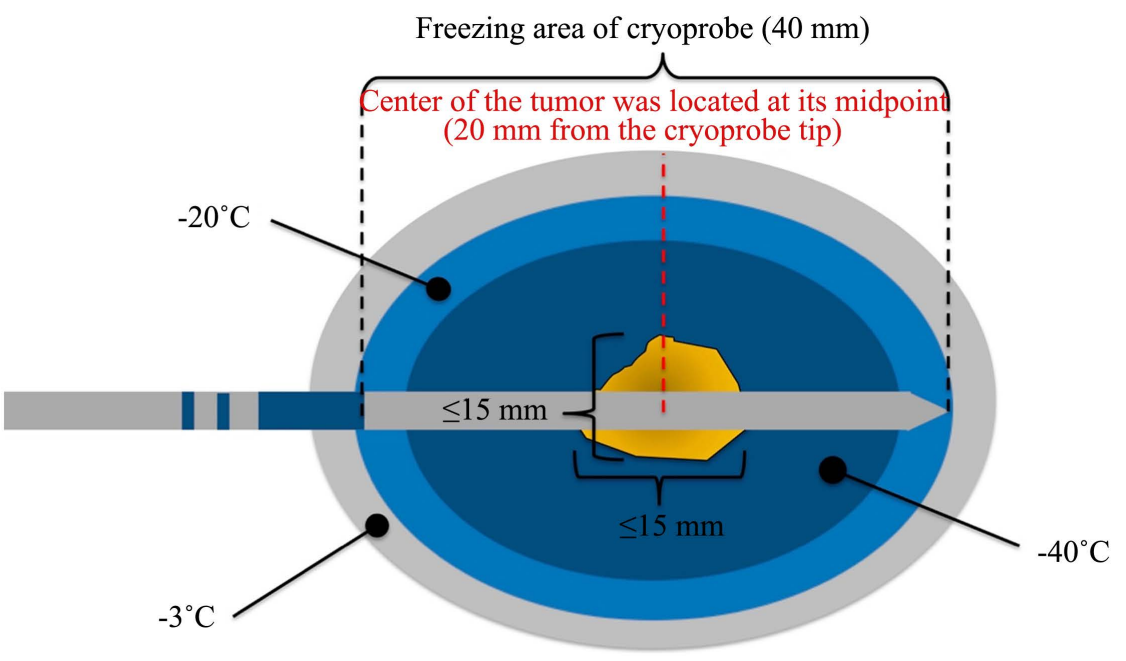

Figure 1. Illustration of cryoablation process. 


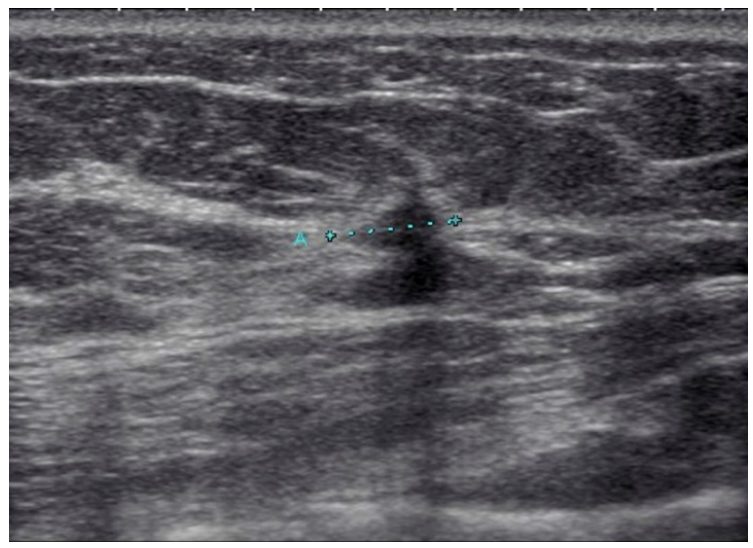

(a)

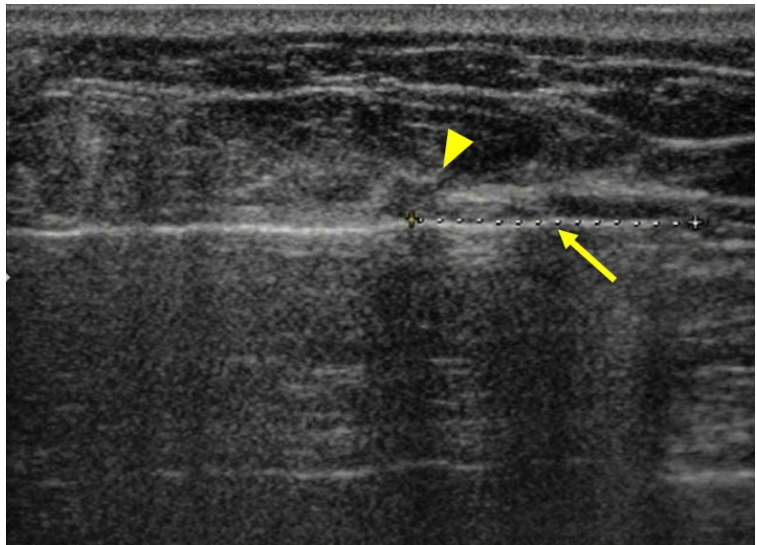

(b)

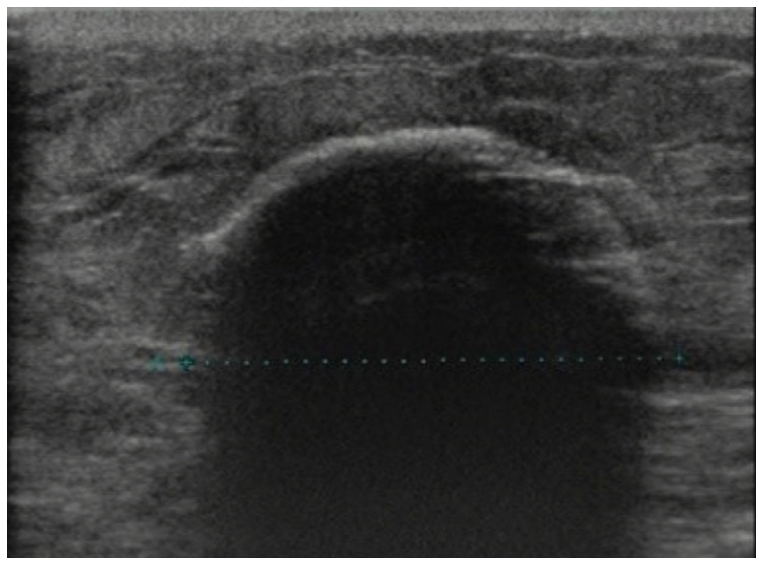

(c)

Figure 2. (a)-(c) Ultrasound visualization of cryoablation process. (a) Ultrasound image of a BC lesion at baseline; (b) The frozen probe (arrow) as it penetrates the tumor (arrowhead) and the appropriate position for the onset of freezing; (c) Ultrasound measurement confirmed that the ice ball has grown to the expected size (per minor diameter of the elliptical ice ball).

System (BD, CR Bard, Inc.)). The ablation area was well seen, and cells were collected from at least three sites: Ablation center and at least two sites of the periphery of spherical ablation area. 
Cosmetic outcomes were recorded at baseline and, then estimated every 6 months after cryoablation using Moiré topography.

\section{Statistical Analysis}

We performed a descriptive analysis of patient- and cryoablation process-related characteristics. Continuous variables were described using median, mean, standard deviation, minimal and maximal values, and percentage. The calculations were performed with Microsoft Excel 2003.

\section{Results}

Total of eight patients (mean age 61.9 years, SD 7.7, ranged 53 - 72 years) underwent ultrasound-guided percutaneous cryoablation as a primary treatment of breast cancer lesions (mean tumor size was $10.3 \mathrm{~mm}$, SD 2.74, ranged 6.8 - 14.5 $\mathrm{mm}$ ). The patients had IDC histology type, estrogen receptor-positive, none of the patients had HER2/neu overexpressing, performance status 0 or 1, estimated per Eastern Cooperative Oncology Group Performance Status Scale (Table 1). The follow-up period ranged from 13 to 34 months with median follow-up of 28 months. Follow up period of seven of eight patients exceeded 24 months, while that of 4 patients (50\%) was 30 months and above. Imaging (US/MG/MRI) did not detect any suspicious areas during the follow-up period. Histopathological analysis (cells collected with VAB at $1-6$ months post-ablation) was performed in seven out of eight cases with no evidence of malignancy (Figure 3(a) and Figure $3(\mathrm{~b})$ ). No residual cancer was seen on core biopsy following the procedure and no recurrences have occurred.

Table 1. Characteristics of the patients at baseline.

\begin{tabular}{|c|c|c|c|c|c|c|c|c|c|c|}
\hline \multirow{2}{*}{ No. } & \multirow{2}{*}{$\begin{array}{l}\text { Age, } \\
\text { years }\end{array}$} & \multirow[b]{2}{*}{ PS } & \multirow{2}{*}{ HT } & \multirow[b]{2}{*}{$\mathrm{ER}, \%$} & \multirow{2}{*}{$\begin{array}{l}\text { PgR, } \\
(\%)\end{array}$} & \multirow{2}{*}{ Her2 } & \multirow{2}{*}{$\begin{array}{c}\text { Ki-67, } \\
\%\end{array}$} & \multicolumn{2}{|c|}{ Tumor characteristics } & \multirow{2}{*}{$\begin{array}{c}\text { SLNB results } \\
\text { (no. of positive } \\
\mathrm{LN} / \text { no. of extracted LN) }\end{array}$} \\
\hline & & & & & & & & $\begin{array}{l}\text { Size at baseline } \\
\text { (per MRI), mm }\end{array}$ & Location & \\
\hline 1 & 66 & 0 & IDC & 99 & 90 & Neg & $1-9$ & 10.59 & Upper outer left & $0 / 3$ \\
\hline 2 & 54 & 0 & IDC & 80 & 30 & Neg & $10-20$ & 6.80 & Upper inner right & $0 / 1$ \\
\hline 3 & 67 & 0 & IDC & 99 & 0 & Neg & $1-9$ & 12.20 & Upper outer right & $0 / 1$ \\
\hline 4 & 70 & 0 & IDC & 99 & 20 & Neg & $10-20$ & 14.49 & Upper inner left & $0 / 1$ \\
\hline 5 & 53 & 0 & $\mathrm{MCN}^{*}$ & 99 & 90 & Neg & $10-20$ & 8.50 & Upper outer right & $0 / 1$ \\
\hline 6 & 55 & 0 & IDC & 99 & 30 & Neg & $1-9$ & 8.90 & Upper mid left & $0 / 1$ \\
\hline 7 & 58 & 0 & IDC & 90 & 100 & Neg & $10-20$ & 13.00 & Lower outer left & $0 / 2$ \\
\hline 8 & 72 & 0 & IDC & 99 & 90 & Neg & $1-9$ & 7.70 & Upper outer right & $0 / 1$ \\
\hline Mean & 61.9 & & & & & & & 10.3 & & \\
\hline $\mathrm{SD}$ & 7.70 & & & & & & & 2.74 & & \\
\hline Min & 53 & & & & & & & 6.8 & & \\
\hline Max & 72 & & & & & & & 14.5 & & \\
\hline
\end{tabular}

PS: Performance status; HT: Histotype; ER: Estrogen receptor; PgR: Progesterone receptor; SLNB: Sentinel lymph node biopsy; IDC: Invasive ductal carcinoma; MCN: Mucinous carcinoma; Neg.: Negative. 


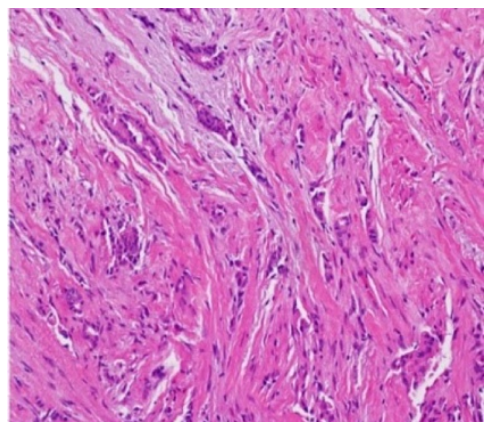

(a)

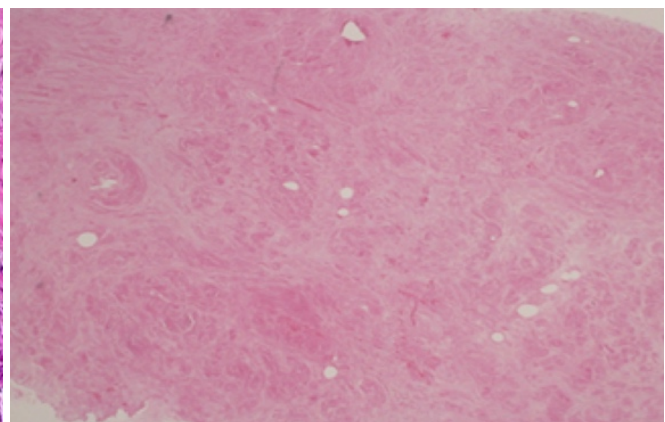

(b)

Figure 3. (a) and (b) Vacuum-Assisted Biopsy (VAB). (a) Invasive carcinoma cell nests in fibroconnective tissue prior to PCA; (b) One-month post-PCA, aspirated tissue biopsy: Collagen fibers and a few adipose tissue areas without lobules, no evidence of residual viable carcinoma cells.

An adverse event was noted: A skin redness 1-week post-cryoablation. The redness resolved spontaneously in about 2 weeks after oral administration of antibiotics and anti-inflammatory analgesics. No other complications or serious adverse events were found (Table 2).

MRI at one-month post-cryotherapy showed various degrees of thermal burns in all patients in the pectoralis major muscle, which were, however, not symptomatic and disappeared after 6 months (Figure 4).

Cosmetic outcomes were assessed visually using Moiré topography at 6-, 12and 24-months and compared with the cosmesis at the baseline. No distortion of nipple position, breast deformity or asymmetry was found. The cosmetic outcomes were estimated as very good (Figure 5(a) and Figure 5(b)).

All patients completed at least one full "freeze-thaw-freeze" cycle of cryoablation. A second "thaw-freeze" cycle was performed in three cases (cases no.1, no.7, and no.8) to obtain the required ablation volume. The mean treatment duration of cryoablation procedures was $42.5 \mathrm{~min}$ (range 33 - $53 \mathrm{~min}$ ), including warming for probe extraction. Iceball was measured during the procedure using ultrasound equipment. The mean size of the ice ball at the end of procedure was $52.7 \times 41.1 \mathrm{~mm}$ (Table 3).

MRI showed spherical zones of necrosis (mean size $54.9 \mathrm{~mm} \pm 12.24 \mathrm{~mm}$, ranged 41.10 - 70.10), consistent with the post-cryoablation healing process. Mean reduction of the post-ablation necrosis area at 6,12 and 24 months, as compared with the previous measurement, were $38 \%(24 \%$ - 54\%), $21 \%(4 \%$ $40 \%)$ and $19 \%(7 \%-37 \%)$ respectively, and overall mean reduction was $58 \%$ (range 42\% - 67\%) after 2 years (Table 4, Figure 6, Figure 7 (a) and Figure $7(\mathrm{~b}))$.

\section{Discussion}

The goal of this work was to evaluate the feasibility and safety of cryoablation as well as describe post-ablation changes in ex-tumor vicinity. We also aimed to validate MRI as tool for post-cryoablation verification of oncologic success with 


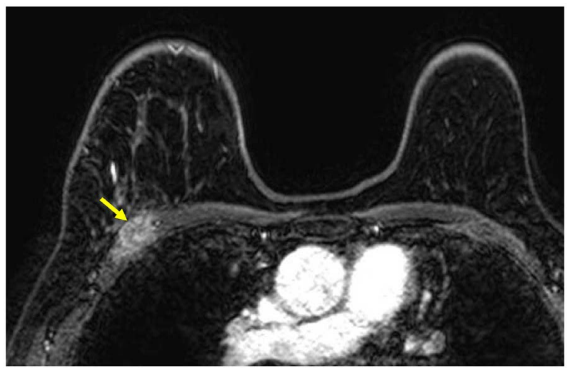

(a)

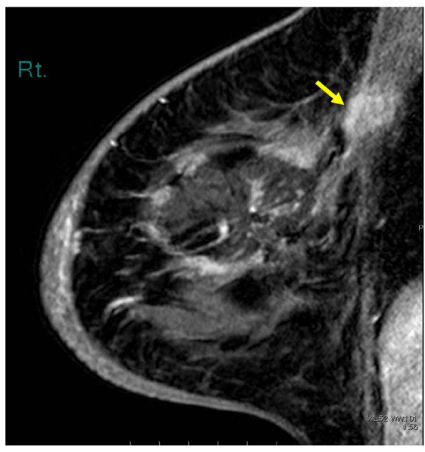

(c)

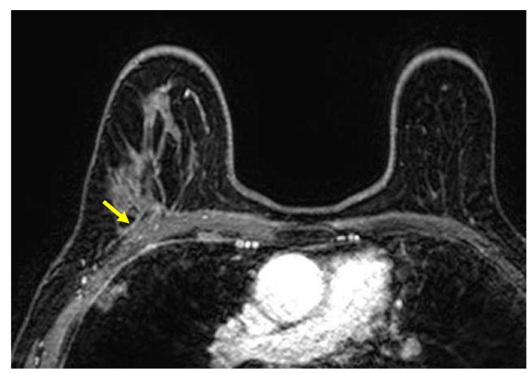

(b)

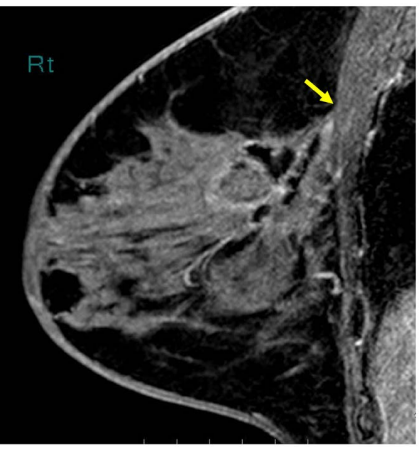

(d)

Figure 4. (a)-(d) Findings of transient muscle freezing injury after PCA on MRI (Case no. 3). (a) One-month post-cryoablation follow-up MRI axial position showing focal enhancement of the pectoralis major muscle (arrow); (b) Six months post-cryoablation follow-up MRI axial position showing no focal enhancement of the pectoralis major muscle (arrow); (c) One-month post-cryoablation follow-up MRI sagittal position showing focal enhancement of the pectoralis major muscle (arrow); (d) Six months post-cryoablation follow-up MRI sagittal position showing no focal enhancement of the pectoralis major muscle (arrow).

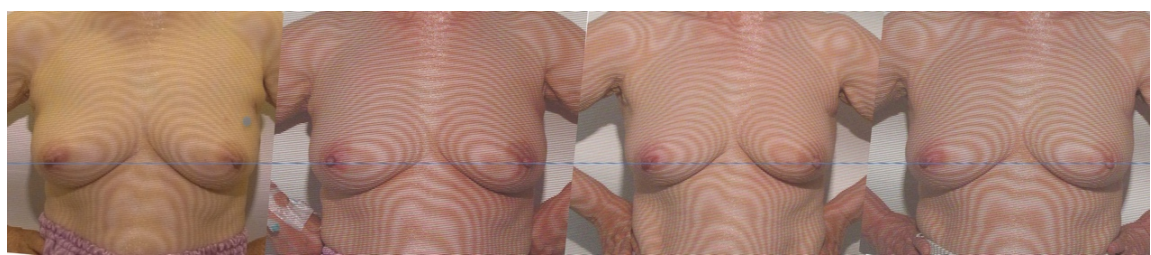

(a)

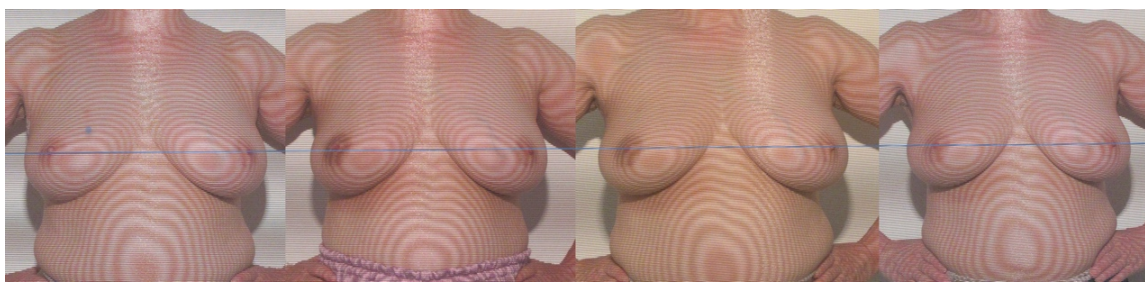

(b)

Figure 5. (a) and (b). Moiré topography used for estimation of cosmetic outcomes. (a) Visualization of Moiré Topography (Case no.1, baseline vs. 6-, 12- and 24-months post-CA). The blue circle on the left upper lateral side at baseline is the site of tumor occupation; (b) Visualization of Moiré Topography (Case no.2, baseline vs. 6-, 12- and 24-months post-CA). The blue circle on the right Upper Inner side at baseline is the site of tumor occupation. 
Table 2. Adverse events.

\begin{tabular}{|c|c|c|c|c|c|}
\hline $\begin{array}{l}\text { Patient } \\
\text { ID }\end{array}$ & $\begin{array}{c}\text { Recurrence } \\
\text { status } \\
\text { (per imaging) }\end{array}$ & $\begin{array}{c}\text { Residual } \\
\text { cancer } \\
\text { (per VAB) }\end{array}$ & $\begin{array}{l}\text { Serious } \\
\text { Adverse } \\
\text { events }\end{array}$ & $\begin{array}{c}\text { Adverse } \\
\text { events }\end{array}$ & $\begin{array}{c}\text { FU time, } \\
\text { months }\end{array}$ \\
\hline 1 & none & none & none & none & 34 \\
\hline 2 & none & none & none & none & 34 \\
\hline 3 & none & none & none & $\begin{array}{c}\text { breast } \\
\text { skin redness }\end{array}$ & 31 \\
\hline 4 & none & none & none & none & 30 \\
\hline 5 & none & none & none & none & 26 \\
\hline 6 & none & none & none & none & 26 \\
\hline 7 & none & none & none & none & 25 \\
\hline 8 & none & none & none & none & 13 \\
\hline Mean & & & & & 27.4 \\
\hline $\mathrm{SD}$ & & & & & 6.36 \\
\hline Min & & & & & 13 \\
\hline $\operatorname{Max}$ & & & & & 34 \\
\hline
\end{tabular}

(mm)

Decrease of post-PCA fat necrosis mass

80

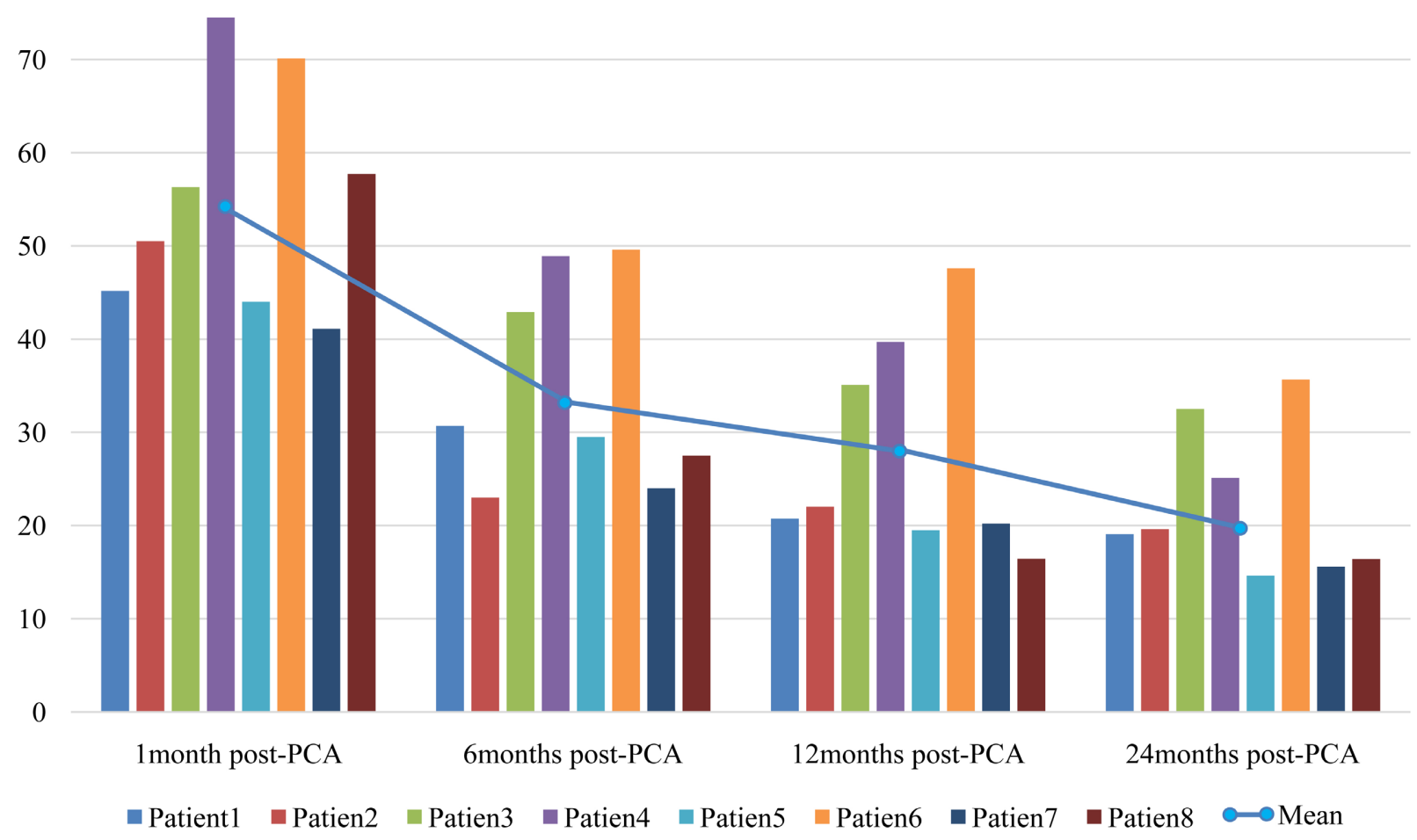

Figure 6. Decrease of post-cryoablation fat necrosis masses. 


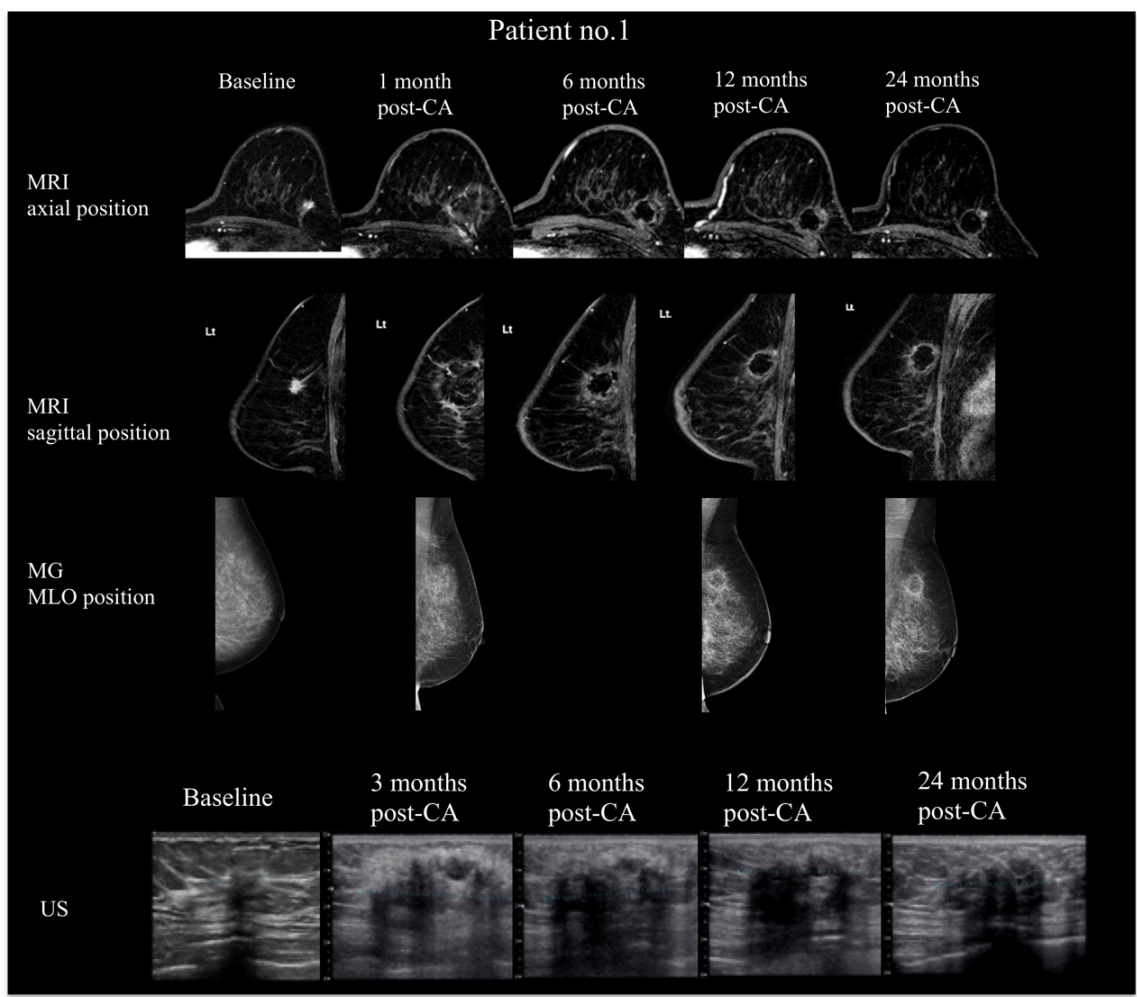

(a)

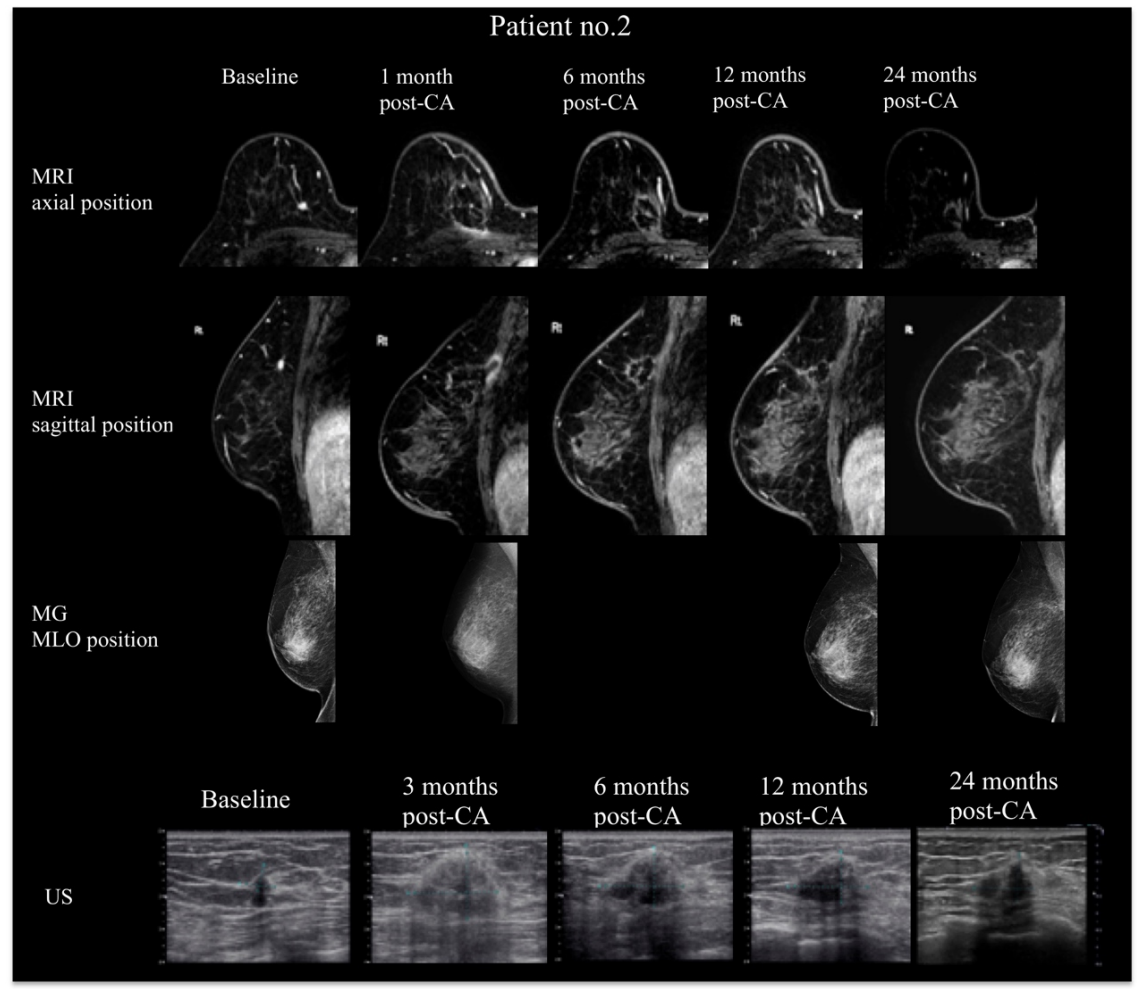

(b)

Figure 7. (a) and (b) Follow up with different modalities. (a) Case no.1: Diminishing of post-ablation necrosis area during follow-up; (b) Case no. 2: Diminishing of post-ablation necrosis area during follow-up. 
Table 3. Characteristics of the cryoablation procedure: freezing duration vs. size of the ice ball.

\begin{tabular}{ccccc}
\hline \multirow{2}{*}{$\begin{array}{c}\text { Patient } \\
\text { ID }\end{array}$} & Number of & Total duration of & \multicolumn{2}{c}{ Freezing zone obtained } \\
\cline { 4 - 5 } & CA cycles & the procedure, min & \multicolumn{2}{c}{ (US-measured) } \\
\cline { 4 - 5 } 1 & 2 & $00: 53: 14$ & 52.8 & 40 \\
2 & 1 & $00: 35: 55$ & 53 & 36.9 \\
3 & 1 & $00: 33: 48$ & 53.9 & 44.7 \\
4 & 1 & $00: 36: 09$ & 51.3 & 43.7 \\
5 & 1 & $00: 34: 54$ & 54.3 & 44.3 \\
6 & 1 & $00: 34: 00$ & 54.2 & 45.8 \\
7 & 1 & $00: 49: 02$ & 54.1 & 41 \\
8 & 2 & $00: 48: 27$ & 48.3 & 34.4 \\
Mean & 1.25 & $00: 40: 41$ & 52.7 & 41.4 \\
SD & & $00: 07: 33$ & 1.9 & 3.8 \\
Min & 1 & $00: 33: 48$ & 48.3 & 34.4 \\
Max & 2 & $00: 53: 14$ & 54.3 & 45.8 \\
\hline
\end{tabular}

Table 4. Decrease of post-cryoablation fat necrosis masses.

\begin{tabular}{ccc}
\hline Patient ID & $\begin{array}{c}\text { Max diameter of fat necrosis mass } \\
\text { one-month post-CA, MRI (mm) }\end{array}$ & $\begin{array}{c}\text { Overall decrease } \\
\text { of fat necrosis mass, \% }\end{array}$ \\
\hline 1 & 45.2 & 19.1 \\
2 & 50.5 & 19.6 \\
3 & 56.3 & 32.5 \\
4 & 74.5 & 25.1 \\
5 & 44.0 & 14.6 \\
6 & 70.1 & 35.7 \\
7 & 41.1 & 15.6 \\
8 & 57.7 & 16.4 \\
Mean & 54.9 & 22.3 \\
SD & 12.24 & 7.47 \\
Min & 41.1 & 7.5 \\
Max & 70.1 & 35.7 \\
\hline
\end{tabular}

vacuum-assisted biopsy. The current pilot study was extremely safe with few minor complications. VAB (performed for 7 out of 8 patients) proved absence of residual disease on biopsy, and no recurrences occurred during follow-up.

In 2021, Fine et al. published the results of 3-year interim analysis of ipsilateral breast tumor recurrence after cryoablation of low-risk early-stage breast cancer (ICE3 Trial) [16]. Earlier, in 2016, the results of ACOSOG Alliance Z1072 
clinical trials were reported by Simmons et al. [11] ICE3 enrolled 194 patients 75 years old on average; mean tumor size was $8.1 \mathrm{~mm}$. Eighty-six participants of ACOSOG trial ( 87 breasts treated) were 62-year-old in average and had a larger mean tumor size of $12 \mathrm{~mm}$. ICE3 protocol included cryoablation alone, without subsequent resection, which enabled to pursue long term Ipsilateral breast tumor recurrence (IBTR) follow up. Breast surgery (mastectomy or lumpectomy) took place within 28 days after cryoablation procedure in the frames of ACOSOG trial; results of pathological analysis of the excised tissue were presented. At 3 years interim analysis (mean follow-up time of 34 months), ICE3 reported Ipsilateral breast tumor recurrence (IBTR) 2.06\% (4/194). According to ACOSOG trial, successful cryoablation was observed in $69 \%$ of the cases (60/87) [11] [16].

Takada et al. publication on PCA-related trials conducted in the last fifteen years present a comprehensive comparative analysis of safety and performance characteristics of cryoablation procedure. Among all studies depicted by Takada, we selected only those which were associated with cryoablation of early-stage breast tumors of size limited by $30 \mathrm{~mm}$ [10]. Tumor size is the pivotal criterion for patients with early-stage breast cancer in favor of a single needle percutaneous cryoablation. The risk of locally incomplete ablation is significantly higher for tumors larger than $2 \mathrm{~cm}$ (Simmons et al.), especially for single-needle ablations [11]. In 2019 Machida et al. published the results of a prospective study, which included 54 patients with early stage of breast cancer (3 DCIS cases included), adjunctive therapies, mean tumor size $8.5 \mathrm{~mm}$. One of 54 cryoablated patients had a recurrence after 54 months of follow-up. The study concluded $98 \%$ of success rate, which is the highest rate in the latest reported prospective studies [17]. In 2017 Pollack et al. published the results of a prospective study in which they used cryoablation to eliminate 20 early-stage breast cancer patients with tumors less than $15 \mathrm{~mm}$. The technical cryoablation success was claimed in all cases, while the absence of malignant cells was achieved in $85 \%$ of participants (post-ablation surgery performed) [18].

In a study of 23 primary breast cancer patients (mean age of 85 years, median tumor size $14 \mathrm{~mm}$, and median follow-up 14.6 months), by Cazzato et al., local tumor control was assessed by comparing baseline and follow-up DCE-MRI and no post-ablation resection was performed. The authors reported that complete cryoablation was recorded in all patients with IDC $<1 \mathrm{~cm}$ or IDC $\leq 1.5 \mathrm{~cm}$ without extensive intraductal components. Five patients recurred during follow-up and two were successfully re-treated with CA. Tumor size was limited by $30 \mathrm{~mm}$. The technical success and local recurrence rates were $100 \%$ and $78.2 \%$, accordingly [12].

In their retrospective study, Machida et al. calculated a level of agreement of two independent observers when reviewing post-cryoablation images. Interobserver agreement ranged from fair to moderate $(0.356-0.434)$. Only $13 \%$ of suspicions findings were identified by both observers in first post-PCA MRI [17].

Ability to timely detect residual malignancy and local recurrence is of upmost 
importance and based on skilled imaging interpretation. Imminent to cryoablation MRI findings may later disappear leaving just a scared tissue but one to two months after cryotherapy it is still quite difficult to differentiate a recurrent lesion from wound healing process. When analyzing post-ablation magnetic resonance images, pace of fat necrosis masses' decrease over time should be considered: In our study, we witnessed a two-fold necrosis' shrinkage in 24 months following the procedure.

We certainly need more trials with a larger number of patients and longer follow-up, not only on feasibility and safety of percutaneous cryo-treatments but although on post-PCA imaging follow-up. Oncologic control may be examined by pathological examination or biopsy, depending on whether post-ablation excision is performed or not. We plan to continue with our prospective research.

\section{Conclusions}

Our study, although conducted on a limited number of patients, showed the feasibility of the cryoablation method for the elimination of small malignant lesions in the breast, along with promising safety prospects.

Percutaneous cryoablation of early-stage low-risk breast cancer tumors smaller than $15 \mathrm{~mm}$ potentially presents a potential substitute for lumpectomy, offering encouraging short- to mid-term oncology results with good cosmesis outcomes. Patients would be able to benefit from local anesthesia in an outpatient setting and a shorter recovery period.

\section{Acknowledgements}

The authors would like to thank Dr. Eisuke Fukuma of Kameda Medical Center and the facility staff for their support in starting the cryosurgery program. We thank USCI Japan Ltd. for support in setting up ProSense ${ }^{\mathrm{TM}}$ System at our center. We are also grateful to all staff of the Breast \& Imaging Center and Clinical Engineers at St. Marianna University School of Medicine for the management of the cryosurgery equipment. Finally, we thank the Data Center members for data management.

\section{Declaration of Funding and Conflicts of Interest}

The authors declare that there is no conflict of interest.

This research received no specific grant from any funding agency in the public, commercial, or not-for-profit sectors.

\section{References}

[1] U.S. Cancer Statistics Working Group (2020) U.S. Cancer Statistics Data Visualizations Tool, based on 2020 submission data (1999-2018): U.S. Department of Health and Human Services, Centers for Disease Control and Prevention and National Cancer Institute. https://www.cdc.gov/cancer/dataviz

[2] NIH (National Institute of Health) (2021) SEER Program (Surveilance, Epidemiology, and the End Results Program). https://seer.cancer.gov/statfacts/html/breast.html 
[3] WHO (2020) GLOBOCAN Report 2020.

https://www.uicc.org/news/globocan-2020-new-global-cancer-data

[4] Matsumoto, K., Haga, K., Kitazawa, T., Seto, K., Fujita, S. and Hasegawa, T. (2015) Cost of Illness of Breast Cancer in Japan: Trends and Future Projections. BMC Research Notes, 8, Article No. 539. https://doi.org/10.1186/s13104-015-1516-y

[5] Siegel, R.L., Miller, K.D., Fuchs, H.E. and Jemal, A. (2021) Cancer Statistics 2021. A Cancer Journal for Clinicians, 71, 7-33. https://doi.org/10.3322/caac.21654

[6] Chu, K.F. and Dupuy, D.E. (2014) Thermal Ablation of Tumors: Biological Mechanisms and Advances in Therapy. Nature Reviews Cancer, 14, 199-208. https://doi.org/10.1038/nrc3672

[7] Pusceddu, C., Paliogiannis, P., Nigri, G. and Fancellu, A. (2019) Cryoablation in the Management of Breast Cancer: Evidence to Date. Breast Cancer, 11, 283-292. https://doi.org/10.2147/BCTT.S197406

[8] Roubidoux, M.A., Yang, W. and Stafford, R.J. (2014) Image-Guided Ablation in Breast Cancer Treatment. Techniques in Vascular \& Interventional Radiology, 17, 49-54. https://doi.org/10.1053/j.tvir.2013.12.008

[9] Pusceddu, C., Sotgia, B., Amucano, G., et al. (2014) Breast Cryoablation in Patients with Bone Metastatic Breast Cancer. Journal of Vascular and Interventional Radiology, 25, 1225-1232. https://doi.org/10.1016/j.jvir.2014.05.001

[10] Takada, M. and Toi, M. (2019) Cryosurgery for Primary Breast Cancers, Its Biological Impact, and Clinical Outcomes. International Journal of Clinical Oncology, 24, 608-613. https://doi.org/10.1007/s10147-019-01448-4

[11] Simmons, R.M., Ballman, K.V., Cox, C., et al. (2016) A Phase II Trial Exploring the Success of Cryoablation Therapy in the Treatment of Invasive Breast Carcinoma: Results from ACOSOG (Alliance) Z1072. Annals of Surgical Oncology, 23, 2438-2445. https://doi.org/10.1245/s10434-016-5275-3

[12] Cazzato, R.L., de Lara, C.T., Buy, X., et al. (2015) Single-Centre Experience with Percutaneous Cryoablation of Breast Cancer in 23 Consecutive Non-Surgical Patients. Cardio Vascular and Interventional Radiology, 38, 1237-1243. https://doi.org/10.1007/s00270-015-1181-5

[13] (2021) Cryoablation of Low-Risk Small Breast Cancer-ICE3 Trial. https://clinicaltrials.gov/ct2/show/NCT02200705

[14] (2021) Cryoablation of Small Breast Tumors in Early-Stage Breast Cancer (FROST). https://clinicaltrials.gov/ct2/show/NCT01992250

[15] (2017) A Phase II Trial Exploring the Success of Cryoablation Therapy in the Treatment of Invasive Breast Carcinoma (ACOSOG Alliance Z1072) Trial. https://clinicaltrials.gov/ct2/show/NCT00723294

[16] Fine, R.E., Gilmore, R.C., Dietz, J.R., et al. (2021) Cryoablation without Excision for Low-Risk Early-Stage Breast Cancer: 3-Year Interim Analysis of Ipsilateral Breast Tumor Recurrence in the ICE3 Trial. Annals of Surgical Oncology, 28, 5525-5534. https://doi.org/10.1245/s10434-021-10501-4

[17] Machida, Y., Shimauchi, A., Igarashi, T. and Fukuma, E. (2019) MRI Findings after Cryoablation of Primary Breast Cancer without Surgical Resection. Academic Radiology, 26, 744-751. https://doi.org/10.1016/j.acra.2018.07.012

[18] Poplack, S.P., Levine, G.M., Henry, L., et al. (2015) A Pilot Study of UltrasoundGuided Cryoablation of Invasive Ductal Carcinomas Up to $15 \mathrm{~mm}$ with MRI Follow-Up and Subsequent Surgical Resection. American Journal of Roentgenology, 204, 1100-1108. https://doi.org/10.2214/AJR.13.12325 